\title{
Téoros
}

Revue de recherche en tourisme

\section{Migration circulaire, tourisme de racines et développement local}

\section{Le cas des migrants duala de France}

\section{Esoh Elamé}

Volume 29, numéro 1, 2010

Tourisme des racines

URI : https://id.erudit.org/iderudit/1024754ar

DOI : https://doi.org/10.7202/1024754ar

Aller au sommaire du numéro

\section{Éditeur(s)}

Université du Québec à Montréal

ISSN

0712-8657 (imprimé)

1923-2705 (numérique)

Découvrir la revue

Citer cet article

Elamé, E. (2010). Migration circulaire, tourisme de racines et développement local : le cas des migrants duala de France. Téoros, 29(1), 46-54.

https://doi.org/10.7202/1024754ar

\section{Résumé de l'article}

L'objet de cette contribution est de montrer l'interaction entre tourisme de racines et développement local en partant de l'observation ethnographique des pratiques touristiques résultant de la migration circulaire des Duala de France en terre ancestrale. Plus concrètement, je vais prouver que les migrants duala en France pratiquent le tourisme de racines, ce qui m'amènera à m'interroger sur l'impact de ce tourisme dans la transmission d'un héritage socioculturel aux générations suivantes, comprenant les enfants arrivés en bas âge et ceux nés en France, et dans le développement local de la ville de Douala.

Je me propose, dans la première partie de ma contribution, de préciser le cadre théorique et méthodologique de ma réflexion. Par la suite, je donnerai une caractérisation du tourisme de racines des migrants duala de France en terre ancestrale et de son impact dans le développement de leur ville d'origine.
Ce document est protégé par la loi sur le droit d'auteur. L'utilisation des services d’Érudit (y compris la reproduction) est assujettie à sa politique d'utilisation que vous pouvez consulter en ligne.

https://apropos.erudit.org/fr/usagers/politique-dutilisation/ 


\title{
Migration circulaire, tourisme de racines et développement local Le cas des migrants duala de France
}

\author{
Esoh ELAMÉ \\ Docteur en Géographie \\ Enseignant-chercheur \\ CIRDFA - Università Cà Foscari (Venise) \\ elame@unive.it
}

\begin{abstract}
RÉSUMÉ: L'objet de cette contribution est de montrer l'interaction entre tourisme de racines et développement local en partant de l'observation ethnographique des pratiques touristiques résultant de la migration circulaire des Duala de France en terre ancestrale. Plus concrètement, je vais prouver que les migrants duala en France pratiquent le tourisme de racines, ce qui m'amènera à m'interroger sur l'impact de ce tourisme dans la transmission d'un héritage socioculturel aux générations suivantes, comprenant les enfants arrivés en bas âge et ceux nés en France, et dans le développement local de la ville de Douala.

Je me propose, dans la première partie de ma contribution, de préciser le cadre théorique et méthodologique de ma réflexion. Par la suite, je donnerai une caractérisation du tourisme de racines des migrants duala de France en terre ancestrale et de son impact dans le développement de leur ville d'origine.
\end{abstract}

Mots-clés: Douala, duala, tourisme de racines, migration circulaire, développement local.

Afin de réfléchir sur l'interaction entre tourisme de racines et développement local, j'ai pris pour terrain d'étude le peuple duala. Il s'agit d'un peuple bantou de la région du littoral du Cameroun ayant pour ancêtres fondateurs Mbèdi et son fils Ewalè (Balandier, 1975; Bureau, 1962 et 1996; Gouellain, 1973; Moumé Etia, 1981 et 1986). Pour rester fidèle à la toponymie traditionnelle camerounaise, j'utiliserai dans ce texte l'orthographe française Douala pour désigner la ville et duala pour nommer le groupe ethnique.

Depuis la période coloniale française au Cameroun, de nombreux Duala migrent chaque année vers la France. Ceux qui sont en situation régulière et leurs descendants n'hésitent pas, quand les conditions financières le leur permettent, de passer un séjour dans les terres ancestrales, seuls ou en famille, afin de renouer les fils de leur généalogie. Pour les descendants de ces migrants, faire ce voyage est un parcours touristique initiatique très symbolique concernant leur identité. Il leur permet de lutter contre le déracinement en découvrant la terre de leurs ancêtres. C'est également pour eux une expérience concrète pour mieux connaître leur pays d'origine à travers son histoire, sa réalité socioculturelle, économique, politique, et pour se faire une mémoire familiale.
Ce qui les amène au-delà des clichés à découvrir in vivo une mosaïque de lieux, de traditions, de manifestations artistiques et de célébrations.

L'observation ethnographique de la migration circulaire des Duala de France et des pratiques touristiques qui en résultent aujourd'hui permet de s'interroger sur le lien existant entre migration circulaire des Duala résidant en France, tourisme des racines et développement local. En quoi les migrants duala en France pratiquent-ils le tourisme de racines et quel est son impact réel dans la lutte contre la pauvreté?

\section{Cadre théorique et méthodologique}

Parler de tourisme de racines des migrants duala en France pour désigner les déplacements qu'effectuent les membres de cette communauté vers la terre de leurs ancêtres semble intéressant pour deux raisons : d'une part, parce que cela offre l'opportunité d'un ancrage identitaire des migrants et de leurs descendants et, d'autre part, parce qu'il entraîne une impulsion économique suivant le schéma classique de l'interaction migration et développement.

La notion de tourisme de racines utilisée ici se définit comme «une forme singulière de circulation à travers 
laquelle les primo-migrants et personnes issues de la migration tentent de se rapprocher physiquement et provisoirement d'un lieu d'origine tenu pour distinct de leur espace résidentiel» (Legrand, 2006 : 165). Sans toutefois glisser dans une vision passéiste, le tourisme de racines fait redécouvrir les traditions, les modes de vie, les saveurs et les objets familiers des aïeux. Producteur et consommateur d'images et de lieux marqués par l'histoire et le mythe, le tourisme de racines, à l'heure des transhumances saisonnières, a la particularité de faire la promotion d'une unité territoriale fondée sur le patrimoine identitaire. Il permet d'aller à la rencontre de sa propre histoire et de son patrimoine matériel et immatériel. Il met ainsi en lien les descendants d'un territoire avec ceux qui font revivre et qui assurent la pérennité de leurs traditions. Afin d'aborder la problématique du tourisme des racines dans une approche postcoloniale strictement en rapport avec les modes de pensée des Noirs d'Afrique, nous ferons référence au tourisme diasporique identitaire des Noirs d'Afrique désigné comme «une forme de circulation dépendant essentiellement des voyages effectués par les migrants originaires de l'Afrique noire vers leur terre d'origine pour honorer des rites et rituels codifiés qui contribuent à façonner leur identité culturelle et le comportement des membres de leur communauté d'appartenance» (Elamé, 2010 : 48). Il s'agit d'un tourisme déclenché par le besoin de respecter un ensemble de pratiques prescrites liées à des croyances magico-religieuses et induisant indirectement des parcours touristiques spécifiques une fois sur place. Dans le cadre de cette étude, j'expliquerai pourquoi chaque voyage touristique d'un migrant duala résidant en France est une occasion de dépaysement total, alternant des périodes d'intenses rencontres humaines entre les pairs, d'immersion ou de réappropriation identitaire, de découvertes ou de réappropriations gustatives et de visites familiales.

La notion de migration circulaire offre aux personnes la possibilité d'exercer une activité (commerce, profession libérale, volontariat ou autre) dans leur pays d'origine tout en conservant leur résidence principale dans le pays d'accueil. Elle concerne différents groupes, par exemple : les gens d'affaires travaillant dans le pays d'accueil et souhaitant démarrer une activité dans leur pays d'origine (ou dans un autre pays tiers); les médecins, les universitaires et d'autres professionnels désireux de soutenir l'économie de leur pays d'origine en y exerçant une partie de leur activité. J'inclurai dans cette définition : tout migrant qui peut, lors de ses vacances ou d'une situation d'urgence, apporter un soutien à sa famille, à sa communauté d'origine ou à son pays; le tourisme de racines des Noirs d'Afrique dans la mesure où les séjours dans le pays d'origine peuvent permettre de concilier questions identitaires et opportunités économiques, ou soutien à la lutte contre la pauvreté au sein de la famille et de la communauté. Les législations sur l'immigration de plusieurs États membres de l'Union européenne (même si les conditions et les situations visées varient d'un État membre à l'autre) contiennent déjà des dispositions qui favorisent la circularité de la migration en permettant à certaines ou à toutes les catégories de ressortissants de pays tiers ayant le statut de résident de quitter le pays pour des périodes déterminées sans perdre ce statut. Cette législation permet aux migrants duala en situation régulière en France de s'offrir, quand l'occasion se présente, une période de tourisme dans les terres ancestrales.

Sur le plan méthodologique, les réflexions émanant du présent article proviennent d'un travail ethnographique. "Contrairement au courant positiviste de recherche qui privilégie davantage les études à grande échelle et l'expérimentation, l'ethnographie s'intéresse aux expériences de vie quotidienne des individus et permet ainsi de mieux comprendre les pratiques sociales existantes.» (GerinLajoie, 2002 : 77). L'ethnographie nous semble en effet très adaptée pour mettre en exergue la complexité du processus de tourisme de racines des migrants duala en tenant particulièrement compte de la construction identitaire qui s'y rattache.

J'ai construit ma contribution à partir de l'observation participante, menée entre 2003 et 2009, de 120 migrants duala résidant en France et engagés dans les pratiques de tourisme de racines. Ma démarche a consisté à observer, décrire, collecter et classer des faits concernant le voyage des migrants duala dans leur ville d'origine. J'ai pour cela réalisé une dizaine de voyages à Douala en cinq ans, chacun d'une durée moyenne de 30 jours. Lors de ces voyages, j'ai eu l'occasion de rencontrer des touristes duala en situation de migration circulaire qui m'ont fait comprendre ce qu'ils pensaient de leur voyage, pourquoi ils s'autorisaient ou non à prendre part à la migration circulaire et le lien qu'ils font entre migration circulaire et tourisme de racines. Ces discussions se sont principalement déroulées dans les familles des migrants, dans les lieux d'agrégation socioculturels, lors des rites traditionnels de mariage, de deuil, dans les lieux de loisirs. J'ai par la suite fait un travail ethnologique consistant à analyser, synthétiser et systématiser les observations résultant de ces discussions. Elles m'ont surtout servies pour initier une théorisation du tourisme de racines des Duala, notamment sa caractérisation. Durant toute la phase de l'enquête ethnographique, je n'ai pas conservé une position et un statut extérieur à la société étudiée de part mes origines ethniques, ma connaissance parfaite des pratiques langagières et des codes culturels duala. En outre, ayant grandi dans le terrain d'étude, je connais plusieurs migrants duala en France. Je suis moi-même en situation de migration circulaire et par conséquent mon expérience personnelle a aussi été d'une très grande utilité pour mieux cadrer ma technique de recherche. Mon immersion dans la vie sociale de la communauté duala en France et au Cameroun, mes contacts relationnels avec les élites traditionnelles et institutionnelles m'ont facilité la collecte des données.

Le travail ici présenté est issu de l'analyse du propos des personnes observées et interrogées. Il est présenté sous forme de discours tenant compte de l'intelligibilité des échanges qui se sont développés avec les sujets observés. La théorisation émanant de cette étude constitue une étape importante dans la recherche de compréhension d'une autre forme de tourisme permettant d'aller au-delà de la perception occidentale de ce phénomène. 


\section{Repérage sociologique et géographique du terrain d'étude}

Les Duala proviennent d'Afrique équatoriale et vraisemblablement de la région congolaise (Balandier, 1975; Gouellain, 1973). Leur migration de la région congolaise les a conduit d'abord dans la vallée de la Sanaga, ensuite dans l'arrière-pays côtier camerounais, plus exactement vers la façade maritime de l'estuaire du Wouri, en repoussant à l'intérieur des terres les Bassa et les Bakoko. Ces derniers, avant le XVII ${ }^{e}$ siècle, occupaient déjà l'espace qui s'appellera plus tard Douala, en souvenir de Ewalè Mbèdi l'ancêtre éponyme des Duala, qu'ils ont accueillis bien avant l'arrivée des Allemands. Spécialisés dans la pêche, activité qu'ils considèrent noble, les Duala vont développer des échanges importants avec d'autres peuples. Plusieurs études (Gouellain,1975; Bureau,1996) ne manquent d'ailleurs pas de souligner que c'est auprès des Bassa et des Bakoko que les femmes duala acquièrent la connaissance de certaines techniques culturales notamment pour le manioc, l'igname et le maïs.

Le peuple duala est segmentaire et s'est construit à travers un processus de conventions lignagères. Dès la génération des petits enfants de l'ancêtre fondateur, les séparations issues de l'affrontement entre aînés et cadets provoquent des conflits qui conduisent à la création de quatre clans, chacun désigné par une appellation où figure le terme bona (qui signifie «les fils de, les descendants de, les gens de») plus le nom de l'ancêtre fondateur. Les quatre clans duala appelés aussi «cantons» sont issus d'une première séparation au début du XIX ${ }^{e}$ siècle des descendants de l'ancêtre fondateur, et d'une deuxième séparation vers les dernières décennies du même siècle. Il s'agit des Bona Njô (dits Bell) et Bona Bèlè Bèlè (dits Bonabèri), puis les Bona Kuo (dits Akwa) et Bona Ebelè (dits Deido). Chaque clan s'est à son tour décomposé en segments suivant une filiation patrilignage accédant ainsi à une autonomie sous contrôle.

Sur le plan organisationnel, le peuple duala est l'unité la plus étendue, appelée tumba (ethnie) en langue duala. Elle est la structure fédératrice des quatre clans, à savoir le canton Bell, le canton Bèlè Bèlè, le canton Akwa, le canton Deido, qui ont chacun à leur tête un chef supérieur. Géographiquement, le territoire où le peuple duala s'est installé, et qui comprend toutes ses structures traditionnelles de gouvernance politique (institutions traditionnelles) et ses espaces magico-religieux, est appelé en langue duala ekombo ou mboa qui signifie le pays, la terre des ancêtres fondateurs, le lieu mythique de l'emprise identitaire, le terroir.

Sur le plan étymologique, Epée (1999: 104) nous apprend que le peuple duala porte rituellement son nom depuis 1578 par la grâce d'Ewalè quand celui-ci installa son peuple au bord du Wouri en un lieu qu'il baptisa péremptoirement Madu M'Ewale ou l'Embouchure d'Ewalè. Madu M'Ewale progressivement simplifié en Madumwale, puis en Madumale, est la forme plurielle de Du l'Ewalè, simplifié en Duwale, qui par une «faute» de transposition Duwal'a Mbèdi est devenu Duala. Ce peuple est à l'origine de la ville-port qui reçoit finalement son nom.

D’une manière générale, les sources orales et les écrits sur les Duala (Wirz, 1973; Brutsch, 1955; Gouellain, 1975;
Kum’à Ndumbè III, 1986; Ralph et Derrick, 1999) montrent bien que nous avons affaire à un peuple conquérant qui a appliqué une politique de domination vis-à-vis des autres peuples de l'hinterland de l'actuel littoral camerounais. Les Duala se sont installés au fond de l'estuaire du Wouri, sur la rive gauche du fleuve, à $24 \mathrm{~km}$ de la mer, dans une vaste zone marécageuse où le dédale de mangroves alentour a toujours été le fief des pêcheurs. Point stratégique de la traite des Noirs jusqu'au milieu du XIX ${ }^{e}$ siècle, ils ont été des intermédiaires privilégiés des Européens sur le commerce des esclaves. Ils ont poursuivis leur hégémonie sur les peuples de l'hinterland du littoral camerounais jusqu'au moment de la colonisation. La position hégémonique des Duala leur a permis de créer d'importantes relations avec l'extérieur, tirant des avantages économiques importants, transformant leurs chefs en « roismarchand» dotés d'une grande expertise dans les fonctions d'intermédiaires. C'est de là qu'ils tirent leur force mais aussi leur faiblesse, car les liens qu'ils entretiennent avec l'extérieur et l'excès d'intermédiation conduisent à la dénaturation de leur personnalité culturelle.

Le peuple duala a marqué l'histoire du Cameroun dans la mesure où les premiers traités avec les Occidentaux furent signés avec leurs chefs. C'est le cas du traité de 1884, signé entre Gustav Nachtigal et les souverains duala de la côte, permettant ainsi à l'Allemagne d'établir son protectorat sur le Cameroun (Owona, 1996; Delancey, 2000; Ngoh, 1996; Njeuma et al., 1989). En 1472, des navigateurs portugais découvrent l'estuaire du fleuve Wouri et voient pulluler dans l'eau des milliers de crevettes. Ils l'appellent Rio dos Camaroes (la rivière des crevettes). Les Duala par déformation linguistique, vont transformer Camaroes, en Cameroun qui désigne aujourd'hui un pays de $475000 \mathrm{~km}^{2}$ (Owona, 1996; Delancey, 2000).

Le contact permanent avec les Occidentaux a aussi facilité une migration des Duala vers l'Europe. Ainsi, lors de la colonisation allemande, une élite autochtone duala fut formée à l'école allemande par les missionnaires pallotins. Plusieurs membres de cette élite bénéficièrent de formations en Allemagne afin d'être utiles dans l'administration de la nouvelle colonie. Le protectorat allemand marque le début officiel des premières migrations de certains membres de l'élite traditionnelle duala vers l'Allemagne. Lorsque éclata la Première Guerre mondiale, en 1914, une avantgarde investit la ville de Douala. En 1915, la région côtière tomba intégralement et, en 1916, les troupes allemandes quittèrent le «Kamerun", qui sera par la suite partagé entre les Français et les Britanniques avant la fin du conflit. En 1919, le traité de Versailles fixant les conditions de la paix entérina le partage franco-britannique du Cameroun (Nkarey, 2006; Njeuma et al., 1989). Les Français, tout comme les Britanniques, prirent initialement possession des régions côtières camerounaises. La France veilla à supprimer toutes les traces de la colonisation allemande. Avec le décret de 1923, elle rendit obligatoire l'enseignement en langue française. Après la Seconde Guerre mondiale, émerge la nécessité de former une élite locale. La préparation de la décolonisation qui aura lieu en 1960, permet à plusieurs jeunes méritants duala, et en particulier à ceux 
appartenant aux familles de notables et à celles plus aisées, d'être alors envoyés en France pour être formés, dans le but de revenir ensuite gouverner le pays. En effet, l'acquisition de titres universitaires et de compétences professionnelles en France était un label important dans la stratégie d'ascension sociale de plusieurs Duala, notamment ceux issus des classes intermédiaires. C'est ainsi que la France devint un lieu de production d'intellectuels, de professionnels, d'ouvriers spécialisés, d'artistes, d'hommes politiques et d'opérateurs économiques duala. Pour la France, cette immigration avait aussi pour finalité de compléter le projet d'assimilation culturelle et de civilisation des soi-disant indigènes duala. Une partie des Duala de la première génération est rentrée avec l'idée de prendre en main l'avenir du Cameroun après l'indépendance. Par la suite, il $\mathrm{y}$ eut une deuxième génération de migrants duala. Elle a surtout été encouragée par l'échec des politiques de développement des décideurs locaux et de celles imposées par le Fonds monétaire international (FMI) qui ont précipité le pays dans l'endettement et la pauvreté. À cela s'ajoute l'absence d'un véritable processus démocratique fondé sur l'alternance de la classe politique, la pluralité idéologique, l'état de droit, la bonne gouvernance et le respect des libertés fondamentales. Aujourd'hui, c'est en France que l'on retrouve la plus importante communauté duala résidant à l'étranger (Okomo Akam, 1997; Kamdem, 2007).

\section{Motivations du tourisme de racines des Duala vivant en France}

Pour mieux comprendre le tourisme de racines de la communauté duala immigrée en France, il faut prendre en compte les représentations que ces migrants se font du retour, en lien avec les pratiques ritualisées qu'imposent leurs traditions. En effet, on peut classer les raisons qui encouragent le tourisme de racines des Duala en deux catégories.

La première catégorie fait référence aux questions magico-religieuses. Dans ce cas, le tourisme de racines est déclenché suite à une obligation de rentrer au bercail sur la terre des ancêtres pour honorer des pratiques prescrites, tels la participation aux funérailles d'un membre de la famille, les rituels de mariage traditionnel, les rites de guérison, etc. (Elamé, $2010: 52$ )

Ainsi, chez les Duala, il faut bien honorer la mémoire des défunts dans la sphère familiale et lignagère. Cela fait partie des pratiques cosmogoniques de leur religion traditionnelle. Les conceptions mortuaires sont extrêmement complexes. Il est en effet admis que les morts ne sont pas morts et qu'ils continuent d'exister sous forme de forces spirituelles et se trouvent ainsi en interaction avec les vivants (Elamé, 2006; Moumé Etia, 1981). De fait, les pratiques funéraires sont une phase importante qui mobilise la famille et la parenté. Chez les Duala, il est admis qu'il n'existe pas de frontière entre le monde visible et le monde invisible, qui, l'un comme l'autre, participent au monde réel. Dans le monde des défunts et des esprits, l'au-delà est le corollaire du monde des vivants de l'ici-bas. Il s'agit de deux mondes distincts, mais tous deux concrets et interdépendants (Elamé, 2006; Dessajan, 2000; Moumé Etia, 1981; Harter, 1968).

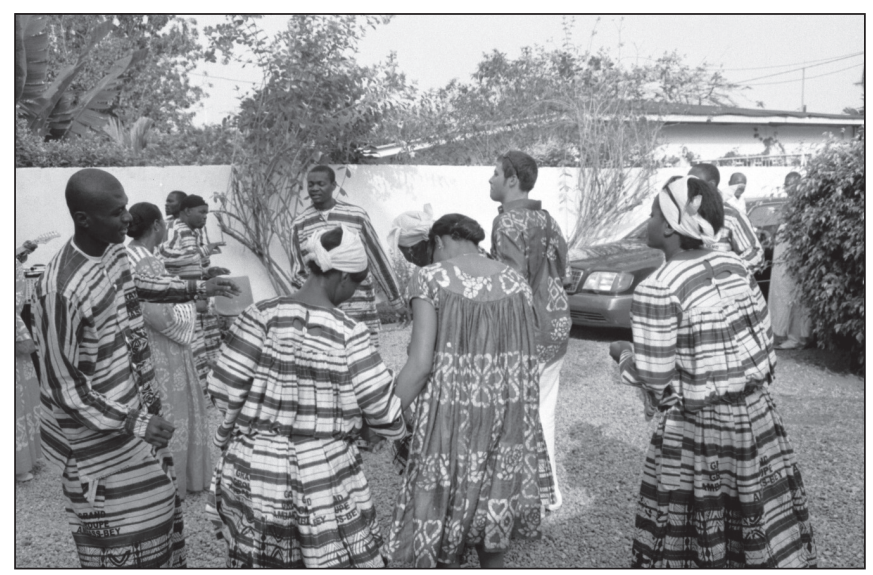

ILLUSTRATION 1 : Moment festif couronnant la fin des cérémonies du mariage traditionnel d'un jeune couple à Douala (photo : Esoh Elamé).

De la même manière, les rituels accompagnant le mariage traditionnel d'un migrant duala en France ou celui d'un membre de la famille peuvent déclencher un tourisme de racines (voir illustration 1). En effet, sur le plan procédural, le mariage traditionnel mobilise tous les membres de la parenté et comprend plusieurs étapes, notamment celle où il faut s'adresser à la famille restreinte (phase appelée «frapper à la porte») et celle de l'ouverture des fiançailles où advient l'acceptation de dons offerts à la famille élargie de la future épouse en suivant une symbolique puisée dans les pratiques ancestrales. Cette phase permet d'obtenir un acte de mariage coutumier qui donnerait implicitement accès au mariage civil.

Les pratiques coutumières et rites de guérison de certaines maladies où la médecine française n'apporte pas de réponse adéquate favorisent aussi le tourisme de racines, car elles interpellent l'univers métaphysique. Dans de tels cas, plusieurs migrants duala sollicitent la médecine traditionnelle où sont pris en compte tous les aspects existentiels de leur être, notamment les relations sociales, le corps dans un sens plus élargi, le psychisme, les émotions. Dans cette démarche, il est admis que le dysfonctionnement du nyolo, le corps, entraîne un déséquilibre global pouvant conduire à des symptômes affectifs, physiques, matériels, immatériels. Chez les Duala, la malchance, Mbea nyolo en langue duala, est une maladie. C'est une détérioration du corps qui se traduit par des perturbations, des malaises psychologiques, et qui, au-delà du symptôme, inclut une recherche de sens. Il s'agit chez les Duala d'une maladie de l'esprit. Il est admis que les guérisseurs traditionnels (Ngambi) puissent intervenir pour enlever la malchance jetée sur un patient comme un mauvais sort et le remettre dans des conditions pour capitaliser ses chances (Elamé, 2010; Rosny, 1992). En clair, la maladie chez les Duala a une signification et une double dimension culturelle et sociale. On ne s'adresse pas à un guérisseur traditionnel pour toutes les maladies. En outre, les traitements traditionnels, contrairement à la conception occidentale moderne de la médecine, permettent de soigner un état qui n’a pas de conséquences physiologiques. 
Le tourisme de racines des migrants duala en France prend donc appui sur une forte demande identitaire de mise en synchronie avec les traditions ancestrales. Contrairement aux autres formes touristiques, il est difficilement programmable. En effet, certains événements comme la mort, la maladie, le mariage traditionnel, un conseil familial à finalité spéciale, l'intronisation d'un chef ne se programment pas. On les subit. L'apparition de ces événements dans la vie migratoire d'un Duala en situation régulière en France montre bien que leur migration n'est pas un aller simple entre un ici et un ailleurs. Ils connaissent assez fréquemment plusieurs retours et surtout plusieurs allers-retours, pour des raisons culturelles ou sociales.

Dans la deuxième catégorie, les descendants des migrants duala de France, notamment leurs enfants et petits-enfants, rentrent plus souvent en terre ancestrale pour des vacances culturelles. Il s'agit de séjours leur permettant de s'imprégner de la culture de leurs parents, d'apprendre la langue, les habitudes culinaires, les pratiques communicationnelles verbales et non verbales, etc. Ce sont des séjours que nous pouvons qualifier à mi-chemin entre l'identitaire et le tourisme de découverte.

\section{Caractérisation du tourisme de racines des Duala vivant en France}

Du point de vue touristique, les déplacements des migrants et de leurs descendants vers et sur la terre de leurs ancêtres est une forme de tourisme en construction s'articulant sur des normes non prescrites provenant de plusieurs champs: culturel, économique, social, et éthique. Il ne s'agit pas d'un tourisme de masse, mais individuel ou de famille, s'organisant dans la plupart des cas suite à une urgence identitaire. Il est donc basé sur des procédures d'optimisation utilisant un cadre procédural qui n'a rien à voir avec le système de pensée européen. Il obéit aux conceptions des Noirs africains de la société, dans le cas des Duala. C'est un tourisme dont les orientations et les normes favorisent l'appropriation concrète par les acteurs locaux du touriste puisque ses caractéristiques sont liées au terroir d'accueil. À partir de l'interprétation des données obtenues de l'observation participante, j'ai dégagé six caractéristiques de base du tourisme de racines chez les migrants duala de France.

La première caractéristique concerne l'accueil. La famille est avisée du voyage et s'organise en conséquence. Le touriste est accueilli à l'aéroport par une délégation toujours importante de membres de sa famille. L'accueil réservé au touriste est aussi fonction du motif principal du voyage. Il peut être très chaleureux, avec des membres de la famille très excités, ou alors émotionnel si le touriste voyage pour participer à un deuil par exemple. Dans tous les cas, le touriste n'est jamais seul à l'aéroport et cela fait partie des codes culturels de la famille élargie. L'accueil donne de l'importance au migrant en situation touristique. Il a le sentiment d'appartenir véritablement à une famille. Le même phénomène se présente lors du retour du touriste : frères, sœurs, oncles, tantes, amis viennent lui dire au revoir à l'aéroport. Leur présence, signe d'affection et élément identitaire, est aussi une sorte de bénédiction pour un bon voyage plein de prospérité. Cela renforce la conviction du migrant sur le fait que bien qu'il parte, il laisse une famille comptant sur lui.

La deuxième caractéristique concerne les infrastructures d'accueil. Ce tourisme tire sa force et son originalité du fait que le touriste est accueilli au sein de sa communauté. La réussite du tourisme de racines des Duala vient du fait que, du point de vue traditionnel, le touriste est accueilli dans sa famille élargie, dans son groupement de base Eboko qui tire son sens de la concession familiale. La famille étendue en tant que groupe unifié par la parenté et la résidence est donc la structure naturelle d'hospitalité du touriste type duala. En plus de la concession familiale, d'autres concessions sont disponibles pour accueillir le touriste. Il s'agit des maisons se référant aux oncles, soit dans la famille patri-centrique (masoso), soit dans la famille matri-centrique (dio) et dans le regroupement de telles cellules (mwèbè). L'articulation de la famille duala, les références de filiation et d'alliance, et la référence spatiale sont au centre des dynamiques d'accueil du touriste. N'étant pas un séjour d'agrément, ni un voyage d'affaires, mais plutôt une visite des membres de la famille pour des raisons identitaires et d'immersion, le cercle familial reste donc le lieu idéal d'accueil. Il permet au migrant de sortir de son quotidien migratoire français, pour se ressourcer. Le touriste retrouve dans son cercle familial, les images de son enfance, les odeurs familières, le rythme de vie ayant animé son enfance. Pour ceux qui sont descendants de migrants duala et qui voyagent pour une immersion culturelle, c'est une occasion exceptionnelle d'apprendre à se familiariser avec certaines pratiques culturelles jusque-là inconnues, et avec des manières de vivre inédites; de nouveaux rythmes de vie et de gestion du temps, etc. La concession familiale au sens large permet de vivre l'historique des lieux et de la culture de ses parents mais aussi de faire la découverte de la différence. Le séjour en famille se vérifie aussi bien dans le cas où le touriste vient pour satisfaire certaines obligations identitaires que dans le cas d'une immersion culturelle pour des descendants de migrants duala. L'existence d'infrastructures hôtelières n'est pas une priorité pour cette forme de tourisme. C'est exceptionnellement que le touriste loge à l'hôtel. De plus en plus de migrants duala, pour témoigner de la réussite sociale de leur projet migratoire, achètent des appartements ou construisent leur maison sur les terrains familiaux qu'ils ont acquis. Ce qui facilite aussi leur venue.

La troisième caractéristique du tourisme de racines des migrants duala concerne la langue de communication. C'est la langue duala ou «Bwambo ba duala», qui appartient au groupe des langues bantoues, qu'il va prioritairement utiliser lors de son séjour pour mieux communiquer avec les membres de sa communauté.

La quatrième caractéristique concerne les aspects culinaires. Le touriste duala, étant accueilli dans la famille élargie, consomme les produits du terroir, les mets traditionnels, préparés suivant les règles gastronomiques duala. Lors des multiples sorties, il pourra lui arriver de manger les mets duala en compagnie de parents ou d'amis, dans certains restaurants du terroir communément appelés «gargotes, circuits, ou chantiers». 
La cinquième caractéristique fait référence à la temporalité en tant qu'objet d'échange et de reconnaissance sociale. Le tourisme de racines des migrants duala tire aussi son importance des systèmes d'échange et de proximité qu'il met en place avec les communautés autochtones. Le touriste est lui-même un autochtone ou un descendant des autochtones. Il rend visite aux membres de sa famille élargie et ces derniers viennent aussi le saluer. Ces rencontres parfois interminables sont des moments importants de discussion et d'échange de points de vue jouant un rôle unificateur où le touriste donne à la notion de temps une dimension d'économie solidaire particulière qu'on ne rencontre pas forcément dans les autres formes de tourisme. Le temps que le touriste duala passe avec les siens pour raconter les événements, évoquer les souvenirs, parler de projets, parfois autour d'une boisson et d'un plat traditionnel, donne à cette forme de tourisme une authenticité fondée sur l'oralité et la solidarité ethnique. L'échange d'idées et de connaissances entre le touriste duala et les membres de sa communauté constitue un bon exemple de réappropriation de sa propre culture. En effet, le touriste se sert de ces moments pour déconstruire certains préjugés, mettre en évidence les bonnes pratiques européennes pouvant servir de levier au développement de sa communauté, parler de développement, des droits de l'homme, de la démocratie, de la bonne gouvernance, etc. Ce sont les moments où le touriste peut facilement s'entretenir avec l'élite intellectuelle, économique et politique de sa communauté pour aborder les questions pertinentes du développement local. À partir de ces échanges, peuvent naître des microprojets de développement permettant aux autochtones d'être acteurs et maîtres d'œuvre d'activités pouvant transformer positivement leur vie. Les expériences menées dans ce sens existent dans les quatre cantons duala où, à partir des migrants en situation touristique, se sont mis en place des projets de développement gérés par les membres des familles, selon les traditions et les rythmes saisonniers locaux. La discussion, les causeries familiales sont des moments importants de renforcement des capacités et de mise en place de projets. Il s'agit donc d'une démarche permettant de construire des «réseaux d'échanges réciproques de savoirs $»$.

La sixième caractéristique concerne l'économie de don associée à cette forme de tourisme. En effet, une démarche économique de don, d'entraide et d'achat équitable fondée sur la relation et la consolidation des rapports caractérise le touriste duala. Lorsqu'il rentre, le touriste duala revient généralement avec des cadeaux pour la parenté. Il est aussi actionnaire de l'entraide familiale dans la mesure où lors de son séjour dans la terre des ancêtres, il prend l'initiative de conclure des engagements pris par le passé vis-à-vis de certains parents. C'est aussi l'occasion de prendre d'autres engagements d'aide avec les membres de la famille, notamment le paiement de la scolarité des plus jeunes frères, cousins et neveux, le financement d'un microprojet économique pour un parent, l'assistance sanitaire à un parent malade, etc. De cette manière, le touriste duala investit significativement dans le renforcement des potentialités et des capacités des ressources humaines de sa famille et devient ainsi un agent concret de lutte contre la pauvreté. Il dépense son argent dans le réseau économique de sa communauté. De par leurs actions, les touristes contribuent à accroître le niveau, la qualité et la pertinence des services de base, particulièrement dans l'éducation, dans la formation et dans la santé en améliorant la qualité et l'accès aux soins ainsi que des actions de sensibilisation et de prévention à l'encontre des personnes à risque. Ils contribuent à un développement urbain équilibré en rénovant le patrimoine bâti familial, en mettant en place des structures de service innovantes qui permettent une meilleure diffusion de la culture, en particulier parmi les jeunes. Ils assurent aussi la promotion du développement économique par l'investissement et l'initiative privée, grâce entre autres au financement et à l'accompagnement de microprojets dans le domaine du commerce et de la PME.

Au-delà de ces six caractéristiques structurelles du tourisme de racines que je viens de mettre en évidence, il est important de souligner d'autres aspects pouvant accompagner et alimenter ce tourisme, notamment le culte des ancêtres, les loisirs et la découverte du Cameroun.

À propos du culte des ancêtres, je signale que lorsque les impératifs identitaires qui déclenchent les voyages en terre ancestrale coïncident avec la première semaine du mois de décembre, période de festivité du culte magico-religieux du Ngondo, de nombreux touristes duala n'hésitent pas à participer aux cérémonies. En effet, le culte de Ngondo est une assemblée traditionnelle réunissant l'ensemble des peuples de la région côtière du Cameroun, depuis maintenant trois siècles. Le culte de Ngondo (Elamé, 2006 : 3) n'est qu'un ensemble d'actions et d'attitudes, agissant sur le mode de vie et de pensée des Duala et des apparentés, leur permettant de perpétuer les croyances et rituels ancestraux en tant que véritables expressions et symboles culturels. Le fait d'être riverains a amené les Duala et les apparentés à développer des pratiques religieuses fortement liées aux forces naturelles aquatiques. Ils considèrent leur patrimoine aquatique comme un bien culturel et spirituel abritant leur divinité du nom de Jengu, appelée aussi communément «Mami Water» ou «esprit de l'eau» par les non-initiés. Reconnu dans la tradition comme une divinité hybride, le Jengu est une représentation mystique de la toute-puissance des esprits de l'eau faisant partie intégrante de la vie des peuples duala. C'est un être aquatique, difforme par rapport aux humains et indissociable de l'eau. Les codes culturels, la manière dont un Duala initié appréhende le monde, part d'une observation de son environnement aquatique. Les aspects cosmogoniques des Duala et des apparentés se manifestent donc par le Jengu donnant lieu à des expressions culturelles particulières les distinguant des autres ethnies. Le Ngondo se compose d'une société secrète, d'un culte et d'un système de croyance ancien, hérité des ancêtres fondateurs (Elamé, 2006 : 3) Il est au centre de plusieurs rites, à vocations spirituelles ou thérapeutiques, non exclusifs les uns des autres. Le culte de Ngondo se fait dans les eaux sacrées du fleuve Wouri et transmet une charge émotionnelle très forte.

Concernant les loisirs, pour se distraire, le touriste duala ne perd pas une occasion de fréquenter certains lieux incontournables de divertissement de son territoire. C'est le cas 
de la fameuse «Rue de la joie» à Deido, avec ses multiples casse-croûte et boîtes de nuit, la discothèque La clé à Akwa, symbole de l'authenticité musicale des Duala et lieu de retrouvailles entre amis. D’une manière générale, chaque canton (Bell, Bèlè Bèlè, Akwa, Deido) dispose de ses structures de loisirs et de gastronomie. Le touriste duala manque rarement l'occasion, lors de ses séjours, de fréquenter les principales plages balnéaires de l'hinterland des Duala et des apparentés, notamment celles de sable noir de Limbé avoisinant les pentes du mont Cameroun et celles de Kribi, au sable fin et doré, bordées de cocotiers, s'égrainant entre la mer et la grande forêt équatoriale. Les merveilleuses chutes de la Lobé de Kribi, se jetant dans l'océan Atlantique en plusieurs cascades dont la plus haute mesure près de $15 \mathrm{~m}$, entraînent des afflux de personnes. Lorsque le voyage se fait durant une période climatiquement favorable, plusieurs touristes identitaires en profitent pour visiter certaines régions du Cameroun. Les zones les plus prisées sont le nord et l'ouest du Cameroun. Au Nord, les touristes duala optent le plus souvent pour la découverte des animaux dans leur cadre naturel (parc de la Bénoué, de Bouba Ndjida, de Waza), les excursions dans des secteurs pittoresques (paysages volcaniques des Kapsiki, villages étagés des monts Mandara), la fréquentation des grands marchés hebdomadaires (Mokolo, Mora, Maroua, Bogo, Pitoa), la rencontre avec les chefs traditionnels des ethnies du Nord (lamidat de Rey-Bouba), la visite aux artisans (brodeurs et artisans du cuir à Maroua, forgerons des monts Mandara, potiers à Guider). À l'Ouest, ce sont les paysages variés des basses latitudes avec les lacs, chutes et reliefs volcaniques qui intéressent les touristes diasporiques duala.

\section{Atouts et limites du tourisme de racines duala}

Le tourisme de racines duala est aujourd'hui une pratique importante de cohésion socioculturelle. Les migrants duala de France, à travers la constante migration circulaire de leur ville de résidence à la terre de leurs ancêtres, contribuent à leur manière au développement local de la ville de Douala et à la cohésion identitaire de leur clan de provenance. Le touriste de racines duala réussit à concilier loisirs, cohésion familiale et enjeux identitaires. Il respecte les critères d'utilité socioculturelle de son voyage en contribuant également à la lutte contre la pauvreté à travers des aides concrètes qu'il va entreprendre pour le compte des membres de sa famille. Le touriste de racines offre des conditions simples et efficaces de soutien aux activités familiales. Il apporte, au cas par cas et selon ses disponibilités économiques, un soutien financier à certains membres les plus marginalisés de sa famille, soit pour financer leurs études ou alors pour la mise en place de micro-activités génératrices de revenus. Cette aide atteint le plus souvent des personnes ne pouvant compter sur aucune autre forme d'aide pour sortir de leur pauvreté. D'une manière générale, l'implication du touriste de racines dans le développement local recouvre trois dimensions complémentaires et indissociables : une dimension socio-économique puisqu'il soutient la mise en place des micro-activités génératrices de revenus aux membres de sa communauté; une dimension éducative dans la mesure où sa présence conduit à des causeries, conversations et discussions avec les membres de sa communauté sur certains problèmes spécifiques tels que le contrôle des naissances, l'éducation des enfants, la promotion de la condition féminine, la promotion de la monogamie, etc.; une dimension culturelle basée sur la perpétuation de l'identité culturelle du peuple duala.

Dans ce scénario, on observe très bien que les populations locales sont au cœur du processus d'accueil du migrant qui organise son séjour en partenariat avec elles : lors de son séjour, le touriste dépense une bonne partie de ses revenus dans les structures économiques locales bénéficiant à la collectivité et permettant de maximiser l'impact sur l'économie locale des territoires d'accueil.

Le tourisme identitaire suscite aussi des initiatives touristiques personnelles permettant aux migrants duala de s'approprier leur passé et de mieux connaître les richesses naturelles et culturelles du Cameroun. Le tourisme de racines apparait donc comme une pratique dans laquelle se superposent de façon particulièrement directe quatre formes d'actions : l'accomplissement de certains devoirs culturels; les retrouvailles familiales permettant de se ressourcer et de maintenir les liens avec la communauté d'appartenance; la fréquentation de certains lieux de divertissement ayant une forte charge émotionnelle, pouvant rappeler pour certains des moments de leur enfance et de leur jeunesse; et enfin la réalisation d'un itinéraire touristique individuel, répondant au schéma classique du tourisme occidental permettant de visiter le Cameroun, de faire des randonnées, d'aller à la plage, etc.

Le touriste de racines duala, comparé aux autres touristes, n'est pas intéressé par l'existence ou non d'une masse critique d'attraits et de services propres à retenir le touriste. Il ne se préoccupe pas de savoir si la terre de ses ancêtres est éloignée ou non des grands pôles touristiques camerounais. Le fait que la terre de ses ancêtres constitue ou pas un produit d'appel fort pour attirer chez elle des flux importants de clientèles touristiques ne l'intéresse pas. Son seul intérêt est sa communauté d'appartenance, sa famille, ses relations amicales. Son choix n'est pas lié à l'existence ou non d'un potentiel touristique à Douala. Comme il a été démontré, une fois sur place il est lui aussi consommateur de l'offre touristique locale, mais sous une forme différente.

Toutefois, le tourisme de racines duala connait aussi des limites qu'il convient de mettre en évidence :

a) Absence d'une politique nationale de promotion du tourisme de racines : L'État camerounais doit s'efforcer aujourd'hui de transformer la diaspora en gisement potentiel de clientèle pour le tourisme. Il est pour cela important de disposer d'une politique visant à encourager le tourisme de racines et d'établir une véritable stratégie de promotion autour de cette action. En ce sens, il faut développer une politique d'action permettant de renforcer le pouvoir des acteurs locaux et non étatiques afin qu'ils répondent aux besoins et attentes des touristes camerounais. Il faut donc favoriser une législation en la matière, responsabiliser les pouvoirs traditionnels, coordonner les activités culturelles du terroir dans une dynamique d'offre territoriale d'événements culturels pouvant profiter au tourisme de racines. 
b) Manque d'itinéraires codifiés de tourisme de racines en mesure de servir de levier à l'identité nationale : on déplore par exemple le fait que le Cameroun ne dispose pas d'actions et d'initiatives sur les routes de l'esclavage et du colonialisme. Si nous prenons le commerce triangulaire, l'histoire des Duala montre bien que ce peuple a pratiqué l'esclavage. Aucune mémoire historique n'existe pour commémorer ce moment tragique de l'histoire des premières nations camerounaises. Pourtant le lancement d'un programme culturel sur la route de la traite atlantique au Cameroun permettrait de faire revivre aux jeunes l'époque où des millions de camerounais furent embarqués de force et condamnés à la servitude dans le Nouveau Monde. Les «routes de la traite», comme on les appelle, partaient aussi de l'intérieur du Cameroun pour aller jusqu'aux marchés des esclaves des côtes camerounaises dont la gestion était assurée par les Duala. La ville de Douala fut en effet une place commerciale importante pour la traite transatlantique. Vendus aux enchères comme du bétail, les captifs descendaient ensuite en file indienne vers le rivage, où les attendaient les bateaux négriers. Aujourd'hui, il n'existe aucun monument rappelant l'inhumanité de ce commerce et signalant à Douala l'endroit où étaient embarqués les esclaves pour le Nouveau Monde. Il en est de même pour la thématique du colonialisme qui manque d'itinéraires de sensibilisation capables d'attirer les voyageurs. Mettre en place des recherches scientifiques sur ces problématiques, organiser des séminaires, des reportages ayant pour finalité de constituer des parcours historiques sur l'esclavage et le colonialisme dans les terres ancestrales des Duala sont indispensables afin de montrer que ce peuple a aussi façonné son histoire par son implication dans ces moments historiques forts, qui marquent l'histoire du Cameroun.

\section{Conclusion}

Le tourisme de racines lié à la migration reste une offre culturelle et commerciale importante réservée aux voyageurs soucieux de redonner un sens identitaire à leur voyage. Son développement devra se faire à l'échelle locale et nationale. Les communautés culturelles camerounaises devraient mieux s'organiser afin de tirer au maximum profit de ce tourisme, en développant des échanges interculturels et en favorisant, dans une démarche éducative, la rencontre entre les migrants voyageurs et les hôtes d'accueil. L'expérience du tourisme de racines des migrants duala résidant en France montre bien que cette forme de tourisme a un impact non seulement culturel, mais aussi économique, notamment dans la lutte contre la pauvreté.

Partant de l'expérience touristique des migrants duala résidant en France, on peut dire que le modèle de développement touristique que le Cameroun devrait envisager doit prendre en compte les opportunités offertes par le tourisme de racines de la diaspora camerounaise à l'étranger. Un processus d'innovation touristique est donc indispensable afin de promouvoir de nouveaux produits touristiques au Cameroun de manière à rendre attrayant le retour au pays d'origine pour de nombreux migrants camerounais à l'étranger. Toutefois, une telle innovation ne peut être efficace que si un travail de fond a été mené pour mieux étudier le phénomène, afin d'identifier les obstacles pouvant empêcher son développement. Il serait pour cela important que l'État camerounais encourage des recherches sur le tourisme de racines. En outre, l'État gagnerait à mettre en place une planification participative impliquant les communautés culturelles et les membres de la diaspora camerounaise à l'étranger pour structurer et faciliter le tourisme de racines. Cela pourra conduire au développement de nouveaux produits de tourisme de racines pour répondre aux exigences spécifiques des migrants camerounais à l'étranger. Pensons par exemple à la mise en place d'un service généalogique informatisé et d'un réseau d'institutions communautaires ayant pour mission de développer et de soutenir la promotion du tourisme de racines au Cameroun.

\section{Bibliographie}

BALANDIER, Georges (1975) «Économie, société et pouvoir chez les Duala anciens », Cahiers d'études africaines, vol. 15, n 59, p. 361-380.

BRUTSCH, Jean René (1955) «Les Traités Camerounais », Études camerounaises, Paris : Publication IFAN, nº 47-48, p. 35.

BUREAU, René (1962) «Ethno-sociologie religieuse des Duala et apparentés ", Recherches et Études camerounaises, Yaoundé, no 7 et 8, 372 p.

BUREAU René (1996) Le peuple du fleuve. Sociologie de la conversion chez les Douala, Paris : Éditions Karthala (coll. Les Afriques). 238 p.

DEHOORNE, Olivier (2002) «Tourisme et migration, entre logiques des marchés et système des mobilités : revue bibliographique», Revue européenne des migrations internationales, vol. $18, \mathrm{n}^{\circ} 1$, mis en ligne le 9 juin 2006, <http://remi.revues.org/index2631.html>, consulté le 10 juillet 2009.

DELANCEY, Mark (2000) « Historical dictionary of the Republic of Cameroon", Lanham : Scarecrow Press, Md, XXXVI. 359 p.

DE ROSNY, Eric de (1992) L'Afrique des guérisons, Paris : Karthala. 223 p.

DESSAJAN, Séverine (2000) «Les Duala et ngondo, ou comment une assemblée traditionnelle permet à un peuple du Cameroun d'affirmer son identité ». Thèse de doctorat, Paris : EHESS. 430 p.

EBOUSSI BOULAGA, Fabien (1976) «L'Identité négro-africaine», Présence Africaine, $\mathrm{n}^{\circ} 99-100$, p. 3-18.

ELAMÉ, Esoh (2006) «La prise en compte du magico-religieux dans les problématiques de développement durable : le cas du Ngondo chez les peuples Sawa du Cameroun ", VertigO - la revue électronique en sciences de l'environnement, vol. $7, \mathrm{n}^{\circ} 3$, mis en ligne le 21 décembre 2006, $<$ http://vertigo.revues.org/2685>, consulté le 5 juillet 2009.

ELAMÉ, Esoh (2010) «Le tourisme diasporique identitaire négro-africain : la communauté Sawa de France», Revue Diaspora, n 14, p. 47-62.

EPÉE, Valère (1999) «Le paradis tabou : autopsie d'une culture assassinée», Douala : Édition CERRAC.

EYELOM, Franklin (2007) L'impact de la première guerre mondiale sur le Cameroun, Paris : L'Harmattan. 198 p.

GERIN-LAJOIE, Diane (2002) «L'approche ethnographique comme méthodologie de recherche dans l'examen du processus de construction identitaire", Canadian modern language review/La Revue Canadienne des langues vivantes, vol. 59, n 1, p. 77-96.

GOUELLAIN, René (1973) «Douala : formation et développement de la ville pendant la colonisation ", Cahiers d'Études africaines, vol XIII, $\mathrm{n}^{\circ} 3$, p. 442-468. 
GOUELLAIN, René (1975) Douala : Ville et histoire, Mémoires de l'Institut d'Ethnologie, 11, Paris : Institut d'Ethnologie, Musée de l'Homme. $411 \mathrm{p}$.

HALL, Michael et WILLIAMS Allan (éd.) (2002) «Tourism and Migration: New Relationships Between Production and Consumption", Geojournal Library, vol. 65, 289 p.

HARTER, Pierre (1968) «Le Ngondo », bulletin de l'Association française pour les recherches et études camerounaises (Faculté des lettres et sciences humaines de Bordeaux), t. 3, p. 61-97.

KAMDEM, Pierre (2007) Les camerounais en Ile de France: Dynamiques migratoires et stratégies d'intégration socio-spatiale différenciées, Paris : L'Harmattan. $314 \mathrm{p}$.

KUM'A NDUMBE III (1986) L'Afrique et l'Allemagne de la colonisation à la coopération : 1884-1986, Douala : Edition Africavenir. 450 p.

LEGRAND, Caroline (2006) «Tourisme des racines et confrontations identitaires dans l'Irlande des migrations », Diasporas, histoire et sociétés, $\mathrm{n}^{\circ} 8$, p. 162-171.

MOUME ETIA, Léopold (1981) Jengu (djengou) : Mammy water, Abbeville, France : Impr. F. Paillart. 32 p.

MOUME ETIA, Léopold (1986) Histoire de Bona Ebele Deido (DoualaCameroun), Douala, $84 \mathrm{p}$.

NGOH, Victor Julius (1996) «History of Cameroon since 1800 », Limbé : Presbook (édition révisée). 367 p.

NJEUMA, Martin (1989) (dir.) Histoire du Cameroun (XIXes. - début $X X^{e}$ s.), Paris : L'Harmattan. $312 \mathrm{p}$.

NKAREY SANTERRE, Jules (2006) Afrique : l'histoire entre le Cameroun anglophone et le Cameroun francophone : de 1472 à 2003, Paris : Publibook. 266 p.

OKOMO AKAM, Suzanne (1997) «La communauté camerounaise en France : étude ethnographique et sociologique». Thèse de Doctorat de Sociologie, Paris : Université de Paris VIII. 442 p.

OWONA, Adalbert (1996) La naissance du Cameroun, 1884-1914, Racines du présent, Paris : L'Harmattan. 229 p.

RALPH, Austen A. (1992) «Tradition, invention et histoire : Le cas du Ngondo », Cahiers d'études africaines, vol. 32, n 126, p. 285-309.

RALPH, Austen A. et Jonathan DERRICK (1999) Middlemen of the Cameroons Rivers: The Duala and their Hinterland, c. 1600-c.1960, Cambridge : Cambridge University Press. 252 p.

UNESCO (2003) Convention pour la sauvegarde du patrimoine culturel immatériel, Paris : Unesco.14 p.

WIRZ, Albert (1973) «La Rivière de Cameroun : commerce précolonial et contrôle du pouvoir en société lignagère ", Revue française d'histoire d'outre-mer, tome LX, n ${ }^{\circ} 219,2^{\mathrm{e}}$ trimestre, p. 190. 